\title{
Conjugation of quantum dots on carbon nanotubes for medical diagnosis and treatment
}

\author{
This article was published in the following Dove Press journal: \\ International Journal of Nanomedicine \\ I March 2013 \\ Number of times this article has been viewed
}

\author{
Seyed Yazdan Madani' \\ Farzad Shabani ${ }^{3}$ \\ Miriam V Dwek ${ }^{2}$ \\ Alexander M Seifalian ${ }^{1,3}$ \\ 'UCL Centre for Nanotechnology \\ and Regenerative Medicine, University \\ College, London, UK; ${ }^{2}$ Department of \\ Molecular and Applied Biosciences, \\ School of Life Sciences, University \\ of Westminster, London, UK; ${ }^{3}$ Royal \\ Free London NHS Foundation Trust \\ Hospital, London, UK
}

\begin{abstract}
Cancer is one of the leading causes of death worldwide and early detection provides the best possible prognosis for cancer patients. Nanotechnology is the branch of engineering that deals with the manipulation of individual atoms and molecules. This area of science has the potential to help identify cancerous cells and to destroy them by various methods such as drug delivery or thermal treatment of cancer. Carbon nanotubes (CNT) and quantum dots (QDs) are the two nanoparticles, which have received considerable interest in view of their application for diagnosis and treatment of cancer. Fluorescent nanoparticles known as QDs are gaining momentum as imaging molecules with life science and clinical applications. Clinically they can be used for localization of cancer cells due to their nano size and ability to penetrate individual cancer cells and high-resolution imaging derived from their narrow emission bands compared with organic dyes. CNTs are of interest to the medical community due to their unique properties such as the ability to deliver drugs to a site of action or convert optical energy into thermal energy. By attaching antibodies that bind specifically to tumor cells, CNTs can navigate to malignant tumors. Once at the tumor site, the CNTs enter into the cancer cells by penetration or endocytosis, allowing drug release, and resulting in specific cancer cell death. Alternatively, CNTs can be exposed to near-infrared light in order to thermally destroy the cancer cells. The amphiphilic nature of CNTs allows them to penetrate the cell membrane and their large surface area (in the order of $2600 \mathrm{~m}^{2} / \mathrm{g}$ ) allows drugs to be loaded into the tube and released once inside the cancer cell. Many research laboratories, including our own, are investigating the conjugation of QDs to CNTs to allow localization of the cancer cells in the patient, by imaging with QDs, and subsequent cell killing, via drug release or thermal treatment. This is an area of huge interest and future research and therapy will focus on the multimodality of nanoparticles. In this review, we seek to explore the biomedical applications of QDs conjugated to CNTs, with a particular emphasis on their use as therapeutic platforms in oncology.
\end{abstract}

Keywords: carbon nanotubes, quantum dots, cancer, photothermal therapy, drug delivery, cytotoxicity, near-infrared light

\section{Introduction}

The diagnosis and treatment of cancer remains a key challenge for biomedical technology. While many classes of anticancer drugs have been discovered and have found widespread use, the majority of conventional chemotherapy treatments are associated with side effects that may include hair loss, nausea, or myocardial infarction. ${ }^{1}$ In addition, there may be an increased risk of infertility, neurotoxicity, nephrotoxicity, vascular toxicity, and thromboembolic complications. ${ }^{2}$ Alongside these issues, other problems associated with conventional chemotherapy include difficulties in clinical administration of drugs, multidrug-resistant tumors, and the inability of the drugs to access the specific
Correspondence: Alexander M Seifalian Professor of Nanotechnology and Regenerative Medicine, University College, London, UK

Tel +44207830290I

Fax +44207472 6444

Email a.seifalian@ucl.ac.uk 
tumor site..$^{2,3}$ Due to the above issues, much research has focused on different approaches toward targeting cancer with drugs that show maximum treatment efficiency with minimum side effects in vivo. ${ }^{3}$ Important factors influencing the design of targeted drug-delivery systems include an efficient means of delivery, preservation of drug-molecular bioactivity, and the enhancement of drug loading and release kinetics toward the drug targets. ${ }^{4}$ Nanomaterials have shown potential in biomedical applications and have received considerable interest in recent years, especially with respect to their future application in the field of cancer diagnosis and cancer treatment. ${ }^{5}$ One of the main factors which make nanoparticles particularly interesting to biomedical science is the size of the materials. This allows them to escape through leaky tumor blood vessels; a feature that results in their accumulation in the parenchyma surrounding tumor blood vessels following systemic injection. ${ }^{6}$ The fastgrowing field of nanotechnology has given rise to the design of new nanomaterials that may circumvent some issues that have been highlighted previously with regard to biocompatibility, and these materials may be used in cancer-related applications. Among the new materials to emerge are carbon nanotubes (CNTs) and quantum dots (QDs), both of which have been the subject of intense investigation with promising results from different in vitro and in vivo approaches for the study of cancer cell metastasis. ${ }^{7,8}$

CNTs are well-ordered, all carbon, hollow graphitic nanomaterials with a high aspect ratio, high surface area and ultralight weight, in addition they contain unique physical and chemical properties. ${ }^{4,5}$ CNTs also absorb near-infrared (NIR) light, generating heat. ${ }^{9}$ These unique properties facilitate the use of CNTs in drug delivery and thermal treatment of cancer. ${ }^{9}$

QDs are semiconductor nanocrystals with superior fluorescent properties with less photobleaching compared with conventional chromophores. ${ }^{6}$ The main application for QDs is for imaging cancer cells, ${ }^{7}$ while CNTs have the ability to destroy cancer cells via thermal ablation and can function as a tool for drug-delivery platforms. ${ }^{8}$ There has been a great interest in the mechanism of cellular uptake of CNTs and different methods have been investigated to elucidate this concept. Labeling CNTs with fluorescence materials such as QDs enables researchers to track the movement of CNTs. The aim of this review is to consider the biomedical applications of conjugated CNTs with QDs for the diagnosis and treatment of cancer.

\section{Quantum dots}

QDs are semiconductor inorganic nanomaterials ranging from $1-10 \mathrm{~nm}$. They contain elements found in groups II-IV (eg, CdSe, CdTe, CdS, and ZnSe) or III-V (eg, InP and InAs) of the periodic table. ${ }^{10}$ Considerable interest has been shown toward these materials in recent years, especially in view of their potential applications in biology and medicine. ${ }^{4}$

A compelling aspect of QDs is their dimensional similarities with biological macromolecules, eg, nucleic acids and proteins. ${ }^{11}$ QDs have fluorescent properties which offer superior features to conventional organic dyes including high quantum yield, ${ }^{12}$ broad absorption, and narrow emission spectra (Figure 1). The properties of these materials include the photostability of coated QDs against photobleaching and tolerance to changes in the $\mathrm{pH}$ of biological electrolytes when compared to fluorescent organic molecules. ${ }^{13}$ QDs can be engineered allowing particle size, shape, and chemical composition to be modulated to suit a given application. Two properties that are often manipulated are the size and composition of QDs; this will determine whether the QD is chemically excited in ultraviolet (UV) or NIR light. For example, nanocrystals of 2-nm size, comprising CdSe, emit light in the range $495-515 \mathrm{~nm}$, whereas larger CdSe nanocrystals of 5-nm size emit light in the range 605-630 nm.

As mentioned earlier, one of the important features of QDs is that they are more photostable than conventional fluorophores, eg, it has been reported that, under the same excitation conditions, $90 \%$ of the fluorescence of a normal organic dye fades within 1 minute, whereas the fluorescence of QDs remains intact even after 30 minutes, ${ }^{14}$ a further feature of these materials is that they may be excited repeatedly. ${ }^{4}$ When both QDs and rhodamine greendextran were exposed to $450 \mathrm{~nm}$ light for 80 minutes, the fluorescence of rhodamine-dextran was lost whilst QDs remained fluorescently stable. ${ }^{15}$ In addition, QDs have a wide excitation spectra and narrow emission spectra compared with conventional dyes. These properties of QDs renders these materials well suited for multiplex imaging. ${ }^{16}$

In terms of the biological application of QDs, promising data have been obtained regarding the toxicity of these

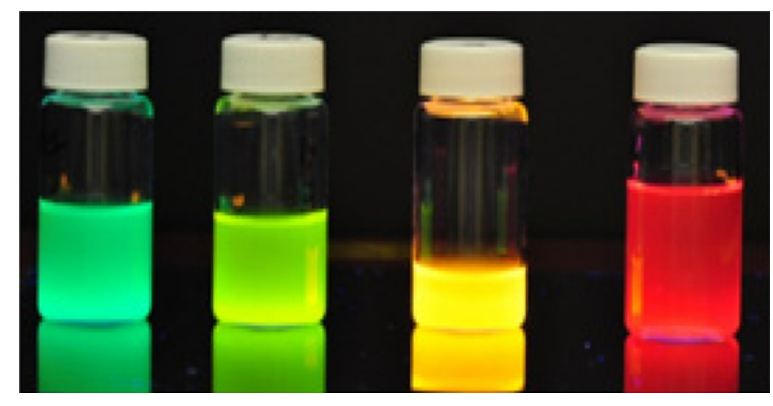

Figure I QDs synthesis in our laboratory. Abbreviation: QDs, quantum dots. 
materials in their surrounding environment. When $10^{8} \mathrm{QDs}$ were injected into zebra fish embryos there was no observed effect on the embryogenesis. ${ }^{17}$ QDs conjugated to tumor cells have been used to study the extravasion step in the metastatic process. In this approach, tumor cells with QDs attached as well as native tumor cells were observed to extravaste into the surrounding tissue in a comparable manner, showing the utility of QDs for monitoring this complex process. ${ }^{14,18}$

\section{Structural design of QDs}

QDs consist of an inorganic core, an inorganic shell and aqueous organic coating. The size of the inorganic core determines the wavelength (color) of light emitted following excitation. An inorganic core consisting of group III-V elements is preferable for clinical work in comparison to group II-IV elements. This is mainly due to the higher stability and lower toxicity of the group III-V elements. Different results by various research groups have indicated that cadmium (in group III-V) is extremely toxic if allowed to leach into the environment and this material also has DNA-damaging properties. Other studies have shown that using cadmium in the cellular environment may lead to the formation of reactive oxygen species, resulting in cell death. ${ }^{19}$ The stability of groups III-V is known to be due to the presence of covalent rather than ionic bonding, ${ }^{20}$ but one of the main disadvantages of group III-V is its low quantum yield in comparison to group II-VI. ${ }^{21}$

The inorganic shell is responsible for increasing the photostability and luminescent properties of the QDs and the aqueous organic coating is used for conjugation of biomolecules to the QD surface ${ }^{22}$ (Figure 2). The photo stability of the inorganic shell has allowed QDs to be used as probes for imaging cells and tissues over long time

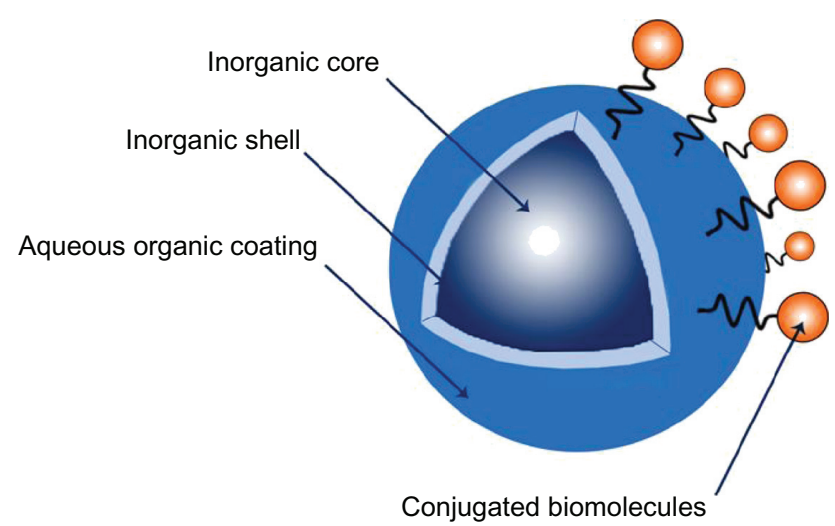

Figure 2 Schematic diagram of QD structure; QDs consist of an inorganic core, an inorganic shell, and aqueous organic coating.

Abbreviation: QDs, quantum dots. periods: a challenging task that necessitates collection of multiple optical sections without damage to the specimen or the aqueous organic coating to which biomolecules are conjugated.

Bimolecular coatings such as the attachment of antibodies enable the delivery of QDs to a specific organ or another site of action. The choice of antibody is important as antibody size may increase the overall size of the quantum dot to between 5-30 $\mathrm{nm}^{23}$ (Figure 3). QDs have been shown to accumulate at disease sites and appear as bright and easily distinguishable stains after illumination, which allows the location of diseased tissue to be identified. ${ }^{15,20}$

While there are many useful features of QDs, a number of issues related to their structure and function still need to be resolved. One of the most problematical is a phenomenon known as "blinking". This is the term used to describe the alternation between the light-emitting and -nonemitting state of the QD. This factor limits the number of photons that can be detected in a given time period and it also contributes to unpredictable photon arrival times. This issue imposes some limitations to the application of QDs and is not fully understood. It has been proposed that this feature of QDs might be suppressed by "passivating" the QDs' surface with thiol moieties, or by using the QDs in free suspension. ${ }^{21}$ Improvement of solubility, stability, and especially the toxicity of the QDs in various environments has also received considerable interest in recent years. ${ }^{19}$ Various groups have shown that factors including size, capping materials, dose, surface chemistry, and coating have direct influence on the above issues, eg, the use of zinc sulfide ( $\mathrm{ZnS}$ ) in the inorganic shell significantly improves the stability of QDs. Coatings

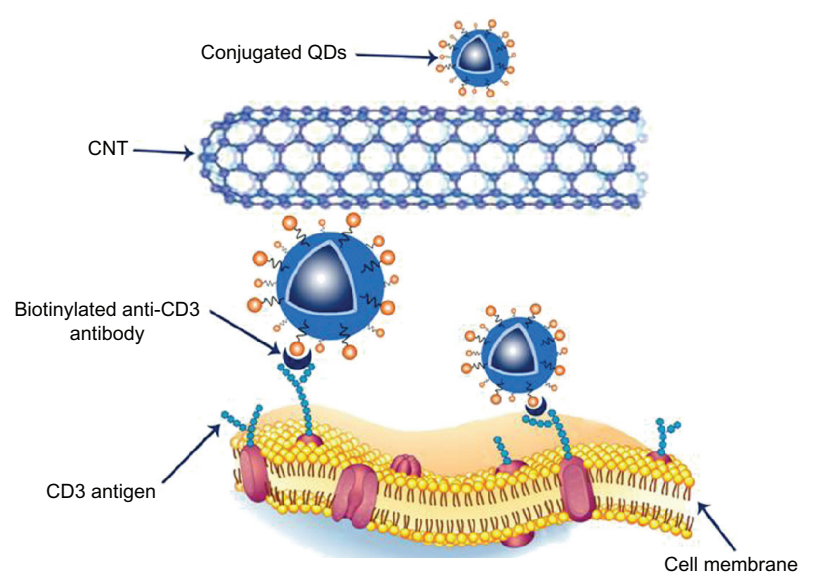

Figure 3 Schematic diagram showing the interaction between $C D 3$ receptors on the Jurkat T leukemia cell membranes and a CNT-QD nanoassembly.

Note: A biotinylated anti-CD3 monoclonal antibody was used to link CD3 to the nanoassembly.

Abbreviations: CNT, carbon nanotube; QDs, quantum dots. 
such as polyhedral oligomeric silsesquioxane-polycarbonate urethane (POSS-PCU) or polyethylene glycol (PEG) ${ }^{24}$ has also been suggested as a method for further improvement of the solubility of QDs. ${ }^{18,19}$

\section{Carbon nanotubes}

CNTs comprise well-ordered carbon atoms with a high aspect ratio, high surface area, ultralight weight, high loading capacity, and high chemical and thermal stability. The synthesis of CNTs can be achieved using several methods including electric arc discharge, laser ablation or catalytic decomposition of hydrocarbons, with each method producing CNTs having differing properties. Nevertheless, these materials have unique structural, electronic, mechanical, and optical properties and potential for application in many fields. ${ }^{25,26}$ More recently, interest in this area has been sparked by the potential for using CNTs as drug-delivery systems for cancer treatment. ${ }^{27}$ In addition, the passive accumulation of CNTs at tumor sites through an enhanced permeability and retention (EPR) effect appears to be a tumor-specific property. ${ }^{25}$

CNTs are available as single-walled (SWCNT) and as multiwalled structures (MWCNT). SWCNTs consist of a single cylindrical carbon layer with a diameter of 0.2 to $2 \mathrm{~nm} ;{ }^{28}$ SWCNTs have a high ratio of length over diameter (aspect ratio) whilst MWCNTs are formed by several concentric layers of rolled graphite with an inner diameter of 1 to $3 \mathrm{~nm}$ and an outer diameter of 2 to $100 \mathrm{~nm} .{ }^{29}$ MWCNTs offer greater potential for drug delivery due to their wider inner diameter in comparison to SWCNTs allowing greater drug loading. It has also been suggested that the outer shell of MWCNTs can be functionalized without damage to the side walls. ${ }^{30}$ There are two main approaches for attachment of drugs to the surface of MWCNTs: covalent and noncovalent attachment; by this process drugs can be incorporated into the interior of the CNT. ${ }^{31}$ In addition to the drug-loading properties of MWCNTs, the chemical and mechanical stability of MWCNTs enables transport of drugs in a range of diverse environments. MWCNTs release substantial vibrational energy after exposure to NIR light resulting in localized heating. This feature of MWCNTs offers potential as an approach to tumor therapy. ${ }^{30}$

In summary, CNTs offer the potential for drug delivery and thermal treatment of tumors whilst QDs offer the potential for tumor imaging. Taken together, these nanomaterial properties offer the opportunity for a compound to be engineered to enable the simultaneous diagnosis and treatment of cancer. $^{7}$

\section{Conjugating CNTs to QDs}

CNTs have a large chemically active surface area; a feature which offers the support of semiconductor nanoparticles, eg, QDs. ${ }^{32}$ The QDs may be linked to the CNT surface by either direct attachment or an intermediate molecule such as a polymer that has previously been conjugated to either the CNT or the QD. Regardless of the approach used, the bonds linking the two nanostructures will be either covalent ${ }^{33}$ or noncovalent. ${ }^{26}$ The conjugation of nanomaterials to the CNT via covalent bonding may be achieved using acid solutions that functionalize the surface of these materials to provide a suitable platform for the CNTs to form covalent bonds with, eg, QDs. An issue with this approach, however, is that the oxidation of the CNTs may be nonuniform, leading to uneven coverage of the surface and in this scenario the nanoparticles tend to attach towards the ends of the CNTs corresponding to the topographical distribution of the functional groups. ${ }^{25}$ In comparison, the noncovalent approach mitigates this concern. ${ }^{34}$ In covalent bonding, a linker between the functional group of the CNTs and QDs is needed. For example, 1-ethyl-3-(3-dimethylaminopropyl) carbodiimide hydrochloride (EDC) may be employed; this simply sticks to the surface allowing weak intermolecular interactions between the CNT and the QDs. ${ }^{35}$ Figure 4 shows QDs coupled covalently to amine and silica functionalized MWCNTs ${ }^{36}$ Whilst direct coupling is a means of conjugating CNTs to QDs, it may be the case that this type of coupling results in quenching of the fluorescence of the QD, and this in turn may result in reduced ability to visualize the QDs. ${ }^{7}$ Another potential issue is partial coverage of the CNTs with QDs. Prefunctionalization of the surface of the CNTs with a polymer-wrapping technique may prove helpful. In this approach, the QDs form bonds with a polymer that coats the CNT side wall. Such a method may also prevent the CNT side wall from invasive damage and defects and also ensures stability of the QDs on the CNT. Various polymers have been used for conjugation of the QDs to CNTs (Table 1). Sodium dodecyl benzene sulfonate (SDBS) has been used as a linker for the conjugation of QDs (in this case $\mathrm{ZnO}$ ) to CNTs in a polar solvent. In this approach the SDBS is prepared in ethanol, forming a micelle, the core of which is hydrophobic (Figure 5). ${ }^{37}$

Another approach is to link 1-pyrene butyric acid N-hydroxysuccinimide esters (PBASE) to the CNTs through $\pi-\pi$ interactions. The QDs form noncovalent bonds to the CNTs coated with PBASE. ${ }^{34}$ A similar experiment was performed using polyethylene imine (PEI) to load the QDs 


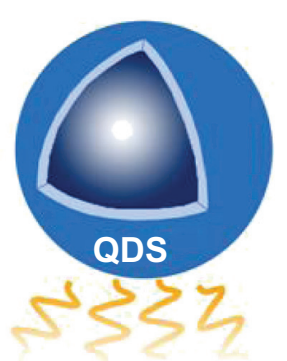

HNH HNH HNH HNH HNH HNH

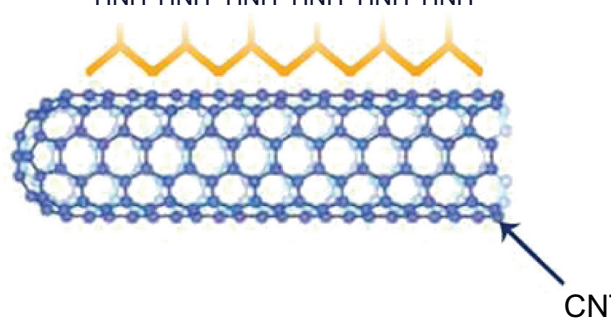

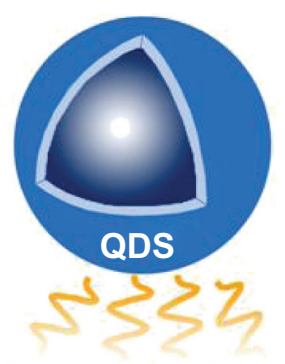

HNH HNH HNH HNHHNH HNH HNH
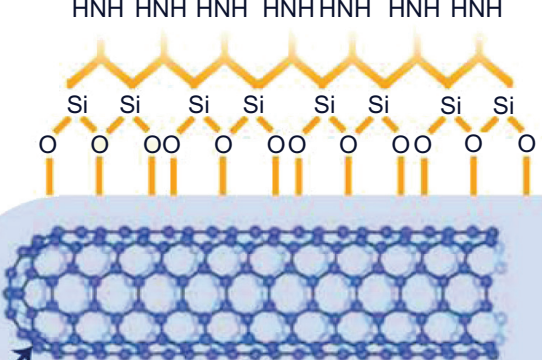

Figure 4 Schematic diagram, showing the conjugation of both QDs to CNT and QDs to CNT coated with $\mathrm{SiO}_{2}$.

Abbreviations: CNT, carbon nanotube; QDs, quantum dots.

onto the surface of CNTs. The high aspect ratio and the "proton-sponge" effect of CNT-PEI, provides a platform for loading QDs onto the surface of CNT-PEI. ${ }^{38}$ Streptavidin, which has a molecular weight of $64 \mathrm{kDa}$, has been used to conjugate QDs to CNTs. The resulting complex was fully dispersed in phosphate-buffered saline; in contrast to the hydrophobic nature of pristine CNTs, these supramolecular nano assemblies were visible by conventional fluorescence microscopy and appear as individual tubes or small rope-like aggregates when viewed by transmission electron microscopy. ${ }^{\text {? }}$

Another novel possibility is to functionalize CNTs with nanocomposite polymers, ${ }^{39}$ for instance, POSS-PCU, ${ }^{40}$ increasing the dispersion of CNTs in biological systems ${ }^{41}$ due to its amphiphilic nature. ${ }^{42}$ POSS-PCU is a thermoplastic that is resistant to degradation ${ }^{43}$ and has anticalcification effects; ${ }^{44}$ it demonstrates superior biocompatibility and is currently being assessed for use in medical implants. ${ }^{45}$ POSS-PCU may be noncovalently bonded to CNTs via $\pi-\pi$ interactions of the aromatic rings of the urea hard segment, which bears stark similarities to the bioconjugation of doxorubicin to CNTs. ${ }^{46}$ The POSS-PCU on the surface of the CNT may then act as a platform for noncovalent conjugation and loading of QDs. ${ }^{22}$ Here at the Department of Nanotechnology and Regenerative Medicine at the University College, London, octa-ammonium-POSS was used as a tool for coating the surface of CNTs. In this experiment, the ammonium group of the octa-ammonium-POSS was initially converted to the amine group by reacting it with $\mathrm{NaOH}$. Conversion of the ammonium groups on the POSS molecule into amine groups allowed for eight functional groups suitable for linkage to the carboxylic acid group (-COOH) on functionalized QDs. Following the treatment of octa-ammonium-POSS with $\mathrm{NaOH}$, the octa-ammonium-POSS was added to the CNT.

Table I Example of the materials used for the attachment of CNT to QDs

\begin{tabular}{|c|c|c|c|}
\hline Type of CNT & Type of QDs & $\begin{array}{l}\text { Material used for } \\
\text { conjugation }\end{array}$ & Final products \\
\hline $\mathrm{MWCNT}^{37}$ & $\mathrm{CdTe}$ & PEI & MWCNT-PEI-CdTe \\
\hline MWCNT $^{36}$ & CdSe & $\mathrm{SiO}_{2}$ & MWCNT-SiO ${ }_{2}-\mathrm{CdSe}$ \\
\hline SWCNT 34 & $\mathrm{CdSe}$ & PBASE & SWCNT-PBASE-CdSe \\
\hline $\mathrm{SWCNT}^{7}$ & $\mathrm{CdSe}$ & Str & SWCNT-Str-CdSe \\
\hline MWCNT ${ }^{37}$ & $\mathrm{CdSe}$ & SDBS & MWCNT-SDBS-CdSe \\
\hline MWCNT $^{35}$ & $\mathrm{CdSe}$ & Mercaptoacetic acid & MWCNT-CdSe \\
\hline
\end{tabular}

Abbreviations: MWCNT, multiwalled carbon nanotubes; SWCNT, single-walled carbon nanotubes; Cdse, cadmium selenide; CdTe, cadmium telluride; PEI, polyethylene imine; $\mathrm{SiO}_{2}$, silica; PBASE, I-pyrene butyric acid N-hydroxysuccinimide ester; QDs, quantum dots; Str, streptavidin; SDBS, sodium dodecyl benzene sulfonate. 


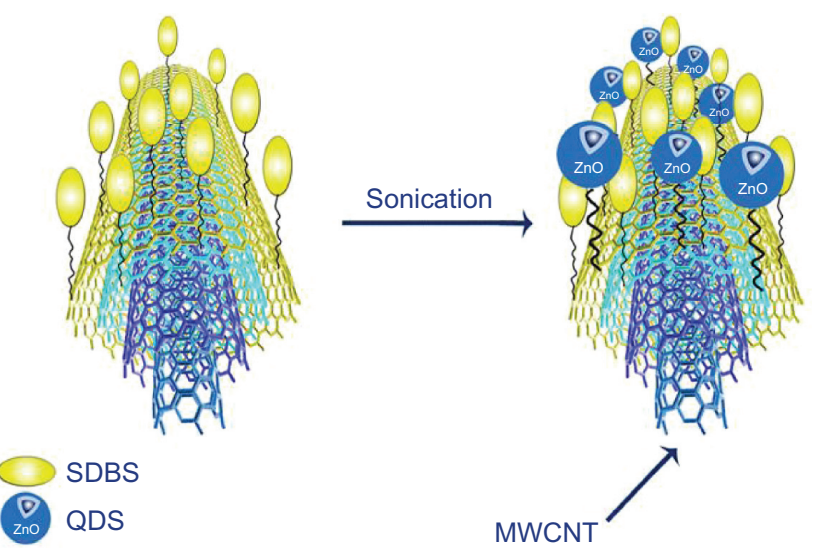

Figure 5 Formation of ZnO QD-MWCNT composites.

Note: Sodium dodecyl benzene sulfonate (SDBS) was used as a linker for conjugation of QDs (in this case $\mathrm{ZnO}$ ) to CNTs in a polar solvent.

Abbreviations: CNTs, carbon nanotubes; MWCNT, multiwalled carbon nanotubes; QDs, quantum dots.

This improved the dispersibility and the biocompatibility of the CNT. In addition, a platform for the uniform attachment of the QDs-COOH on the CNT's surface was formed. Our finding showed that the attachment of the QDs to the CNT generated a more intense fluorescence emission spectra in comparison to the direct attachment approach.

\section{Factors that influence conjugation of CNTs with QDs}

The size of CNT is an important factor that determines the location of attachment of QDs to the CNT. When CNTs are treated with acid (eg, $\mathrm{HNO}_{3}[70 \%] / \mathrm{H}_{2} \mathrm{SO}_{4}[95 \%-98 \%]$ ) for extended periods of time, the majority of CNTs exhibit reduced segment size. Also, it has been suggested that for very short nanotubes, the likelihood of sidewall attachment of QDs as opposed to end-binding of QDs is reduced. As the size of the CNT decreases, so the number of QDs that tend to form bonds at the end of the CNT increases. For the QD to attach to the end of the CNT, the tubes need to be roughly $200 \mathrm{~nm}$ long ensuring that no acid group is attached to the side walls. Conjugation of the QD to the CNTs may be achieved in three different ways: (i) via QD clustering at one end of a CNT; (ii) via QD clustering at both ends of a CNT; and (iii) via a QD cluster "sandwich" between two CNTs.

The distance between the QD and the CNT influences quenching of the QD; ${ }^{32}$ the closer the QD to the CNT, the less QD fluorescence. QD size also influences the quenching of QDs; smaller QDs have a higher surface-to-volume ratio and therefore for smaller QDs, the quenching is increased. The concentration of the CNTs plays a role in the luminescent intensity of QDs; a gradual decrease in photoluminescent intensity of QD-CNT conjugates has been reported when the
CNT concentration increases from 50 to $200 \mathrm{mg} / \mathrm{L} .{ }^{37}$ Once QDs are attached to CNTs, the energy transfer between the conjugated species and the CNT occurs in the photoexcited state. In the excited state, the electrons from the donor are transferred to the acceptor via channels through long-range dipole-dipole interactions; this results in the attenuation of the photoluminescent emission of the QDs. ${ }^{37}$ The factors that could influence transfer of electrons, are spectral overlap integral, center-to-center distance and relative orientation of donor and acceptor. ${ }^{32}$

\section{Methods for characterization of CNT-QDs complex}

The most commonly used methods to verify the conjugation of QDs to CNTs are atomic force microscopy and transmission electronic microscopy ${ }^{25}$ (Figure 6). Another method that
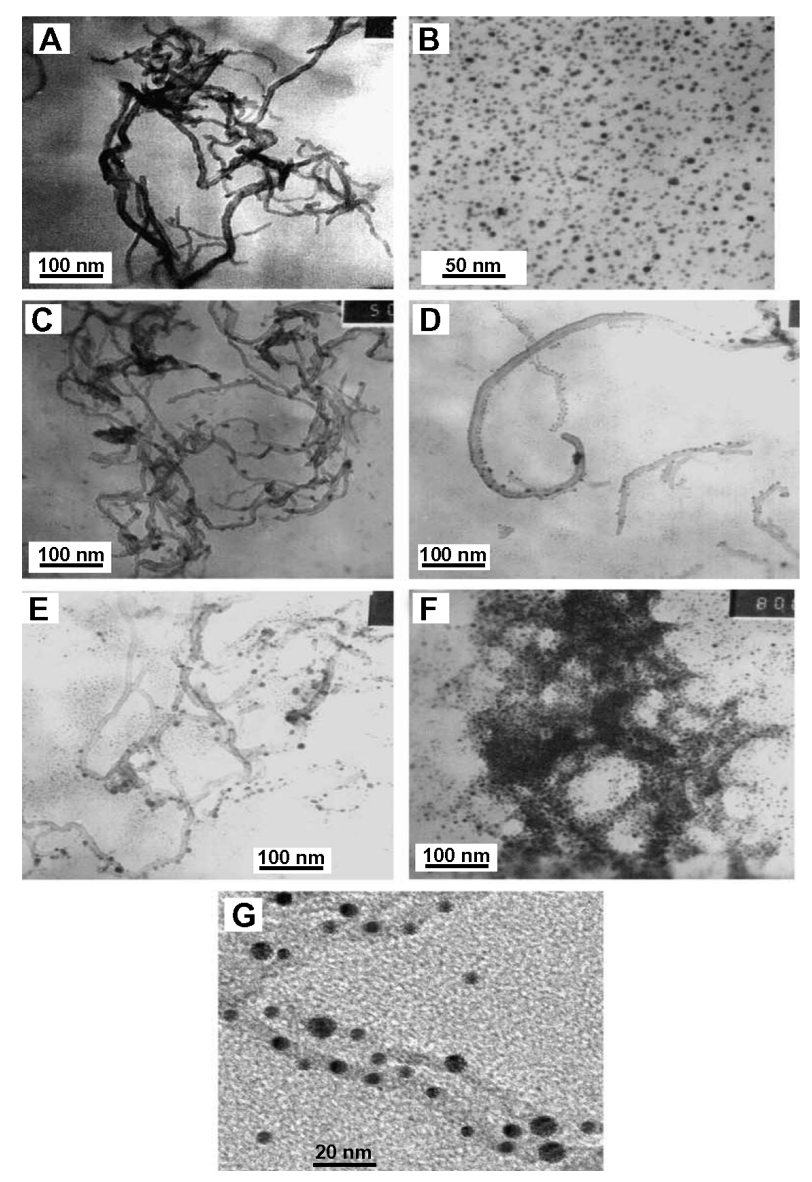

Figure 6 Transmission electron micrographs (TEM) illustrating (A) MWCNT-NH (B) 2.8-4.6 nm CdSe QDs; (C) mixture of MWCNT-COCl and QDs-COOH; (D) QDs/MWCNT = I:10; (E) QDs/MWCNT = I:I; (F) QDs/MWCNT = 10:1; (G) high-resolution TEM image of QDs-MWCNT (QDs/MWCNT = I:I).

Note: Copyright $\odot$ 2006. Reprinted with permission from Elsevier. Pan B, Cui D, He R, Gao F, Zhang Y. Covalent attachment of quantum dot on carbon nanotubes. Chem Phys Lett. 2006;417(4-6):419-424.35

Abbreviations: Cdse, cadmium selenide; MWCNT, multiwalled carbon nanotubes; QDs, quantum dots. 
could be used to confirm the attachment of CNTs to QDs is Fourier transform-infrared (FTIR). ${ }^{37}$

In an experiment, the FTIR technique was used to investigate the attachment of QDs to CNTs. Oxidized MWCNTs give spectral peaks at $1644 \mathrm{~cm}^{-1}, 1704 \mathrm{~cm}^{-1}$, and $3403 \mathrm{~cm}^{-1}$, which is consistent with the characteristic peaks of carboxylic acid and phenolic groups on acid treatment of MWCNTs. However once MWCNTs are conjugated to QDs, new peaks appear at $1653 \mathrm{~cm}^{-1}, 2977 \mathrm{~cm}^{-1}$, and $3411 \mathrm{~cm}^{-1}$; these correspond to $\mathrm{C}=\mathrm{O}-, \mathrm{C}-\mathrm{H}-$, and $\mathrm{N}-\mathrm{H}$-stretching modes of amide and may be used to determine the formation of MWCNT-QDs conjugated through amide bonds. ${ }^{47}$

Another method used to investigate structural and electronic change following coupling of QDs to CNTs was Raman spectroscopy. ${ }^{37}$ UV-VIS spectroscopy may also be used to check the stability of QD-CNT conjugates in aqueous solutions. Using this approach, QD-CNT conjugates have been observed to be stable for up to 7 weeks. ${ }^{35}$

\section{The effect of concentration of QDs on conjugation to CNTs}

Increasing the concentration of QDs affects their spatial arrangement on the surface of the CNTs. When the quantity of QDs to be linked to SWCNTs is increased so they become more uniformly distributed across the entire surface $(15,25$, and $50 \mu \mathrm{M})$ and this is associated with a reduction in aggregate formation, however, the QD loading must be determined for each experimental condition. $^{37}$

\section{Conjugation of CNTs to QDs and the effect on the UV-VIS absorption spectra}

The absorption spectrum of pure MWCNTs and those coated with $\mathrm{SiO}_{2}$ are similar in that they are featureless, with a higher degree of scattering in the MWCNTs coated with $\mathrm{SiO}_{2}$. When the CNTs are attached to QDs minor changes to the spectra are observed, however, upon the conjugation of QDs to CNT, precoated with silica, the composites retain their luminescence spectra with intense absorption peaks observed. ${ }^{36}$

\section{Biomedical applications of CNTs conjugated to QDs}

The QD-CNT complex has applications in engineering through to biomedical sciences. Massive developments have been taking place in the usage of QD-CNT complexes in the optoelectronic and biosensor fields. ${ }^{48}$ The recent industrial usage of QD-CNT complexes includes the areas of computer science, electronics, and mechanical engineering. ${ }^{49}$

The conjugation of QDs to CNT can result in novel hybrid nanocomposite materials displaying unique physical and biological properties. ${ }^{50}$ Due to the electrochemical luminescent properties of QD-CNT complexes, ${ }^{51}$ one of the major applications of these materials is intracellular fluorescent imaging. ${ }^{22}$ Furthermore, QD-CNT complexes can also function as biosensors and biological nanoprobes as well as tools for drug delivery into cells. ${ }^{52}$

In terms of their application as nanoprobes, one of the main advantages of these materials in comparison to organic fluorophores is their ability to be observed with highresolution electron microscopy. ${ }^{53}$ In contrast to spherical nanoparticles, longer nanotubes have a larger inner volume, which allows the CNT to be filled with anticancer drugs, ${ }^{54}$ potentially enabling CNTs to deliver anticancer drugs to a given site. However in order to target the drug-loaded CNTs to the site of action, the surface of these materials need to be modified, preferably with the use of an antibody or a peptide (eg, anti-HER2 of the tubes to cancer cells). For example, since the number of folic acid receptors on the surface of the cancer cells increases, the presence of folic acid on CNT allows the tubes to be used to target cancer cells and enhance drug delivery. ${ }^{55}$ CNTs loaded with the anticancer drugs are injected into the circulation so that the antibody on the surface of the CNTs would direct these materials to the site of action. ${ }^{56}$ The drugs contained within the CNT are delivered to the cells depending on stimulation factors such as a change in the $\mathrm{pH}$ or by an enzyme produced by the tumor that may cleave the drug molecule and release them from the nanotube. Identifying the location of CNTs pre- and postdrug delivery is currently the main aim of researchers working in this area. Combining QDs to CNTs may enable the CNT to be located to particular cell types and has been shown not to be a barrier to penetration into inaccessible tumor sites. In addition, the QD-CNT complex is less likely to be recognized by macrophages and minimizing engulfment would prolong the blood circulation, enhancing the drug-delivery process. In addition, it has recently been shown that QD-CNT complexes conjugated to drugs does not affect their pharmacological properties. ${ }^{10}$ By attaching different QDs to CNTs containing different drugs, the delivery of drugs to cancer cells could be monitored, which allows the efficacy of treatments to be evaluated. ${ }^{57}$ Although there have been many suggestions with regards to the conjugation of QDs to CNTs and their application in the area of drug delivery there is limited understanding of the limitations of this approach. The attachment of QDs to the surface of CNTs 
limits spaces for the attachment of the anticancer drugs to the CNT surface. ${ }^{54}$

\section{Limitations of CNTs conjugated to QDs in vivo}

Once a large particle enters the vasculature, it is likely to be removed through the reticuloendothelial system, however, if the size of the particles is small enough ie, on a nanoscale $(<100 \mathrm{~nm})$ they would bypass this process. When CNTs are coated with QDs, their diameter exceeds $200 \mathrm{~nm}$, which renders them potentially immunogenic and likely to be transported for destruction in the liver or spleen. In order to overcome this problem, SWCNTs have been used in place of MWCNTs. SWCNTs have a diameter in the range of 1 to $3 \mathrm{~nm}$ whereas the diameter of the MWCNT are in the range of 70 to $150 \mathrm{~nm}$. Smaller QDs may also be synthesized thereby reducing the final diameter of the construct. ${ }^{8}$ Another major problem is attenuation of the photoluminescent properties of QDs: upon conjugation to CNTs, the photoluminescence of QDs-MWCNT has been reported to be decreased by $50 \%$ in comparison to the photoluminescence of QDs alone. ${ }^{34}$ Increasing the concentration of functionalized CNT results in increased quenching of QDs, the effect is most prominent for $\mathrm{CNT}_{2} \mathrm{NH}_{2}$, less pronounced for CNT-OH, and weakest for CNT-COOH. Despite the above findings, a recent study showed minimum changes to the quenching of QDs upon conjugation to MWCNT. ${ }^{58}$

\section{Directing nanoparticles to cancer cells}

Research is ongoing in an attempt to understand the important factors and to establish systems for successful uptake of nanoparticles into cancer cells. ${ }^{10}$ The entry of nanomaterials into the cell requires transit across the nanometer-thin lipid bilayer. This may occur through a range of mechanisms, eg, via endocytosis or receptor-mediated uptake, which are also known as active or passive transport mechanism. The latter would rely on adequate surface functionalization and passive targeting is much slower and less efficient than active targeting. When passive targeting is used, QDs accumulate in tumor cells due to their enhanced membrane permeability and the retention effect. For nanomaterials to be useful in a clinical setting, they must demonstrate the following basic requirements: (1) minimal aggregation during long-term storage; (2) water solubility; (3) retain their functionality; and (4) be biocompatible with respect to the target.

\section{Conclusion and future directions}

The emergence of nanotechnology in biology and medicine has garnered significant attention and interest in recent years.
The novel properties of CNTs and QDs make them particularly attractive candidates in experimental nanobiology. The progression from the laboratory bench to clinical bedside largely depends on elucidating the mechanism of action of these nanoparticles and its interaction with the human immune system. Nevertheless, the potential for CNTs and QDs to function as multimodal nanotheranostic agents would likely pave the way for future treatment designs. Using CNTs conjugated with QDs has opened doors to a new generation of scientific research that has the potential to revolutionize the future of medicine.

\section{Acknowledgments}

The authors acknowledge the financial support of Javon Charitable Trust, London, UK for research and development work on detection and treatment of cancer using nanoparticles.

\section{Disclosure}

The authors report no conflicts of interest in this work.

\section{References}

1. Chaudhary UB, Haldas JR. Long-term complications of chemotherapy for germ cell tumours. Drugs. 2003;63(15):1565-1577.

2. Batra R, Davies JN, Wheatley D. Extensive arterial and venous thrombo-embolism with chemotherapy for testicular cancer: a case report. Cases J. 2009;2:9082.

3. Moghimi SM, Hunter AC, Murray JC. Long-circulating and targetspecific nanoparticles: theory to practice. Pharmacol Rev. 2001;53(2): 283-318.

4. Lay CL, Liu HQ, Tan HR, Liu Y. Delivery of paclitaxel by physically loading onto poly(ethylene glycol) (PEG)-graft-carbon nanotubes for potent cancer therapeutics. Nanotechnology. 2010;21(6):065101.

5. Jamieson T, Bakhshi R, Petrova D, Pocock R, Imani M, Seifalian AM. Biological applications of quantum dots. Biomaterials. 2007;28(31): 4717-4732.

6. Yaghini E, Seifalian AM, MacRobert AJ. Quantum dots and their potential biomedical applications in photosensitization for photodynamic therapy. Nanomedicine (Lond). 2009;4(3):353-363.

7. Ghasemi Y, Peymani P, Afifi S. Quantum dot: magic nanoparticle for imaging, detection and targeting. Acta Biomed. 2009;80(2):156-165.

8. Klingeler R, Hampel S, Buchner B. Carbon nanotube based biomedical agents for heating, temperature sensoring and drug delivery. Int $J$ Hyperthermia. 2008;24(6):496-505.

9. Madani SY, Tan A, Dwek M, Seifalian AM. Functionalization of single-walled carbon nanotubes and their binding to cancer cells. Int $J$ Nanomedicine. 2012;7:905-914.

10. Mansur HS. Quantum dots and nanocomposites. Wiley Interdiscip Rev Nanomed Nanobiotechnol. 2010;2(2):113-129.

11. Chan WC, Maxwell DJ, Gao X, Bailey RE, Han M, Nie S. Luminescent quantum dots for multiplexed biological detection and imaging. Curr Opin Biotechnol. 2002;13(1):40-46.

12. Bae PK, Kim KN, Lee SJ, Chang HJ, Lee CK, Park JK. The modification of quantum dot probes used for the targeted imaging of his-tagged fusion proteins. Biomaterials. 2009;30(5):836-842.

13. Chan WC, Nie S. Quantum dot bioconjugates for ultrasensitive nonisotopic detection. Science. 1998;281(5385):2016-2018.

14. Iga AM, Robertson JH, Winslet MC, Seifalian AM. Clinical potential of quantum dots. J Biomed Biotechnol. 2007;2007(10):76087. 
15. Dubertret B, Skourides P, Norris DJ, Noireaux V, Brivanlou AH, Libchaber A. In vivo imaging of quantum dots encapsulated in phospholipid micelles. Science. 2002;298(5599):1759-1762.

16. Han M, Gao X, Su JZ, Nie S. Quantum-dot-tagged microbeads for multiplexed optical coding of biomolecules. Nat Biotechnol. 2001;19(7):631-635.

17. Rieger S, Kulkarni RP, Darcy D, Fraser SE, Koster RW. Quantum dots are powerful multipurpose vital labeling agents in zebrafish embryos. Dev Dyn. 2005;234(3):670-681.

18. Gabizon A, Martin F. Polyethylene glycol-coated (pegylated) liposomal doxorubicin. Rationale for use in solid tumours. Drugs. 1997; 54 Suppl 4:15-21.

19. Ghaderi S, Ramesh B, Seifalian AM. Fluorescence nanoparticles "quantum dots" as drug delivery system and their toxicity: a review. J Drug Target. 2011;19(7):475-486.

20. Bharali DJ, Lucey DW, Jayakumar H, Pudavar HE, Prasad PN. Folate-receptor-mediated delivery of InP quantum dots for bioimaging using confocal and two-photon microscopy.JAm Chem Soc. 2005;127(32): 11364-11371.

21. Manna L, Scher EC, Li LS, Alivisatos AP. Epitaxial growth and photochemical annealing of graded $\mathrm{CdS} / \mathrm{ZnS}$ shells on colloidal CdSe nanorods. J Am Chem Soc. 2002;124(24):7136-7145.

22. Tan A, Yildirimer L, Rajadas J, De La PH, Pastorin G, Seifalian A. Quantum dots and carbon nanotubes in oncology: a review on emerging theranostic applications in nanomedicine. Nanomedicine (Lond). 2011;6(6):1101-1114.

23. Jiang W, Papa E, Fischer H, Mardyani S, Chan WC. Semiconductor quantum dots as contrast agents for whole animal imaging. Trends Biotechnol. 2004;22(12):607-609.

24. Locatelli E, Ori G, Fournelle M, Lemor R, Montorsi M, Comes FM. Click chemistry for the assembly of gold nanorods and silver nanoparticles. Chemistry. 2011;17(33):9052-9056.

25. Banerjee $\mathrm{S}$, Wong SS. In situ quantum dot growth on multiwalled carbon nanotubes. J Am Chem Soc. 2003;125(34):10342-10350.

26. Georgakilas V, Gournis D, Tzitzios V, Pasquato L, Guldi DM, Prato M. Decorating carbon nanotubes with metal or semiconductor nanoparticles. J Mater Chem. 2007;17(26):2679-2694.

27. de la ZA, Gambhir SS. Drug delivery: keeping tabs on nanocarriers. Nat Nanotechnol. 2007;2(12):745-746.

28. Klumpp C, Kostarelos K, Prato M, Bianco A. Functionalized carbon nanotubes as emerging nanovectors for the delivery of therapeutics. Biochim Biophys Acta. 2006;1758(3):404-412.

29. Bekyarova E, Ni Y, Malarkey EB, et al. Applications of carbon nanotubes in biotechnology and biomedicine. J Biomed Nanotechnol. 2005;1(1):3-17.

30. Madani SY, Naderi N, Dissanayake O, Tan A, Seifalian AM. A new era of cancer treatment: carbon nanotubes as drug delivery tools. Int $J$ Nanomedicine. 2011;6:2963-2979.

31. Arsawang U, Saengsawang O, Rungrotmongkol T, et al. How do carbon nanotubes serve as carriers for gemcitabine transport in a drug delivery system? J Mol Graph Model. 2011;29(5):591-596.

32. Haremza JM, Hahn MA, Krauss TD, Chen S, Calcines J. Attachment of single CdSe nanocrystals to individual single-walled carbon nanotubes. Nano Lett. 2002;2(11):1253-1258.

33. Maneeprakorn W, Malik MA, O'Brien P. Developing chemical strategies for the assembly of nanoparticles into mesoscopic objects. J Am Chem Soc. 2010;132(6):1780-1781.

34. Landi BJ, Evans CM, Worman JJ, Castro SL, Bailey SG, Raffaelle RP. Noncovalent attachment of CdSe quantum dots to single wall carbon nanotubes. Mater Lett. 2006;60(29-30):3502-3506.

35. Pan B, Cui D, He R, Gao F, Zhang Y. Covalent attachment of quantum dot on carbon nanotubes. Chem Phys Lett. 2006;417(4-6):419-424.

36. Olek M, Busgen T, Hilgendorff M, Giersig M. Quantum dot modified multiwall carbon nanotubes. J Phys Chem. 2006;110:12901-12904.

37. Dutta M, Jana S, Basak D. Quenching of photoluminescence in $\mathrm{ZnO}$ QDs decorating multiwalled carbon nanotubes. Chemphyschem. 2010;11(8):1774-1779.
38. Jia N, Lian Q, Tian Z, et al. Decorating multi-walled carbon nanotubes with quantum dots for construction of multi-color fluorescent nanoprobes. Nanotechnology. 2010;21(4):045606.

39. Raghunath J, Zhang H, Edirisinghe MJ, Darbyshire A, Butler PE, Seifalian AM. A new biodegradable nanocomposite based on polyhedral oligomeric silsesquioxane nanocages: cytocompatibility and investigation into electrohydrodynamic jet fabrication techniques for tissue-engineered scaffolds. Biotechnol Appl Biochem. 2009;52(Pt 1):1-8.

40. Bakhshi R, Edirisinghe MJ, Darbyshire A, Ahmad Z, Seifalian AM. Electrohydrodynamic jetting behaviour of polyhedral oligomeric silsesquioxane nanocomposite. J Biomater Appl. 2009;23(4): 293-309.

41. Cousins BG, Das AK, Sharma R, et al. Enzyme-activated surfactants for dispersion of carbon nanotubes. Small. 2009;5(5):587-590.

42. Kannan RY, Salacinski HJ, Butler PE, Seifalian AM. Polyhedral oligomeric silsesquioxane nanocomposites: the next generation material for biomedical applications. Acc Chem Res. 2005;38(11):879-884.

43. Ghanbari H, Cousins BG, Seifalian AM. A nanocage for nanomedicine: polyhedral oligomeric silsesquioxane (POSS). Macromol Rapid Commun. 2011;32:1032-1046.

44. Ghanbari H, Kidane AG, Burriesci G, Ramesh B, Darbyshire A, Seifalian AM. The anti-calcification potential of a silsesquioxane nanocomposite polymer under in vitro conditions: potential material for synthetic leaflet heart valve. Acta Biomater. 2010;6(11): $4249-4260$

45. Ghanbari H, de Mel A, Seifalian AM. Cardiovascular application of polyhedral oligomeric silsesquioxane nanomaterials: a glimpse into prospective horizons. Int J Nanomedicine. 2011;6:775-786.

46. Liu Z, Sun X, Nakayama-Ratchford N, Dai H. Supramolecular chemistry on water-soluble carbon nanotubes for drug loading and delivery. ACS Nano. 2007;1(1):50-56.

47. Ravindran S, Bozhilov KN, Ozkan CS. Self assembly of ordered artificial solids of semiconducting $\mathrm{ZnS}$ capped CdSe nanoparticles at carbon nanotube ends. Carbon. 2004;42(8-9):1537-1542.

48. Cui D, Pan B, Zhang H, et al. Self-assembly of quantum dots and carbon nanotubes for ultrasensitive DNA and antigen detection. Anal Chem. 2008;80(21):7996-8001.

49. Li F, Son DI, Cho SH, Kim WT, Kim TW. Flexible photovoltaic cells fabricated utilizing $\mathrm{ZnO}$ quantum dot/carbon nanotube heterojunctions. Nanotechnology. 2009;20(15):155202.

50. Fei Q, Xiao D, Zhang Z, Huan Y, Feng G. A novel silica-coated multiwall carbon nanotube with CdTe quantum dots nanocomposite. Spectrochim Acta A Mol Biomol Spectrosc. 2009;74(2):597-601.

51. Jie G, Li L, Chen C, Xuan J, Zhu JJ. Enhanced electrochemiluminescence of CdSe quantum dots composited with CNTs and PDDA for sensitive immunoassay. Biosens Bioelectron. 2009;24(11):3352-3358.

52. Gao X, Cui Y, Levenson RM, Chung LW, Nie S. In vivo cancer targeting and imaging with semiconductor quantum dots. Nat Biotechnol. 2004; 22(8):969-976.

53. Michalet X, Pinaud FF, Bentolila LA, et al. Quantum dots for live cells, in vivo imaging, and diagnostics. Science. 2005;307(5709):538-544.

54. Mitchell DT, Lee SB, Trofin L, et al. Smart nanotubes for bioseparations and biocatalysis. J Am Chem Soc. 2002;124(40):11864-11865.

55. Xiao Y, Gao X, Taratula O, et al. Anti-HER2 IgY antibody-functionalized single-walled carbon nanotubes for detection and selective destruction of breast cancer cells. BMC Cancer. 2009;9:351.

56. Prajapati VK, Awasthi K, Gautam S, et al. Targeted killing of Leishmania donovani in vivo and in vitro with amphotericin $\mathrm{B}$ attached to functionalized carbon nanotubes. J Antimicrob Chemother. 2011; 66(4):874-879.

57. de la Zerda A, Gambhir SS. Drug delivery: keeping tabs on nanocarriers. Nat Nanotechnol. 2007;2(12):745-746.

58. Zhang Y, Qin W, Tang H, et al. Efficient assembly of multi-walled carbon nanotube-CdSe/ZnS quantum dot hybrids with high biocompatibility and fluorescence property. Colloids Surf B Biointerfaces. 2011; 87(2):346-352. 


\section{Publish your work in this journal}

The International Journal of Nanomedicine is an international, peerreviewed journal focusing on the application of nanotechnology in diagnostics, therapeutics, and drug delivery systems throughout the biomedical field. This journal is indexed on PubMed Central, MedLine, CAS, SciSearch $\AA$, Current Contents ${ }^{\circledR} /$ Clinical Medicine,

Journal Citation Reports/Science Edition, EMBase, Scopus and the Elsevier Bibliographic databases. The manuscript management system is completely online and includes a very quick and fair peer-review system, which is all easy to use. Visit http://www.dovepress.com/ testimonials.php to read real quotes from published authors.

Submit your manuscript here: http://www.dovepress.com/international-journal-of-nanomedicine-journal 